\title{
El trabajo del hogar en el Perú: transiciones laborales, educación y ciclo económico*
}

\author{
Cecilia Garavito**
}

\begin{abstract}
RESUMEN
El objetivo de este trabajo es analizar las transiciones desde el trabajo del hogar remunerado hacia otras ocupaciones, el desempleo y la inactividad, así como los determinantes de estas transiciones. Para ello empleamos los datos panel de la ENAHO para los periodos 1998-2002, 2002-2006 y 2007-2011. Luego de un análisis detallado de las transiciones parciales y totales de cada panel, encontramos que alrededor de un tercio de las trabajadoras del hogar se quedan en la misma ocupación y que un porcentaje similar pasa a al trabajo independiente o a ocupaciones asalariadas de baja productividad. Al estimar los determinantes de las transiciones desde el trabajo del hogar remunerado hacia otras ocupaciones y hacia la inactividad, encontramos que un mayor nivel de educación está asociado a una salida hacia otra ocupación, si bien en el caso de las trabajadoras del hogar usualmente es hacia el trabajo independiente. Finalmente, las transiciones desde el trabajo del hogar remunerado hacia otros empleos o hacia la inactividad dependen del ciclo económico, si bien los efectos marginales son menores que en el caso de las mujeres en general.
\end{abstract}

Palabras clave: mercado de trabajo, trabajo del hogar remunerado, transiciones laborales, oferta de trabajo, género.

Clasificación JEL: J15, J16, J21, J46

\section{Housework in Peru: Working Transitions, Education and Economic Cycle}

\begin{abstract}
The aim of this work is to analyze the transitions from paid housework toward other occupations, unemployment and inactivity, and the determinants of these transitions. We use the panel data from the ENAHO for the periods 1998-2002, 2002-2006, and 2007-2011. After a detailed descriptive analysis of partial and complete panels, we find that around one third of the paid house-workers stay in this occupation while a similar percentage moves to independent work or low productivity salaried work. After estimating the transitions from paid housework to other occupations, and to inactivity, we find that a higher level of education is always related

* Este trabajo es una versión mejorada del documento de trabajo escrito como parte del periodo de investigación que la Pontificia Universidad Católica me otorgó durante tres semestres académicos. La versión preliminar se puede encontrar aquí: http://files.pucp.edu.pe/departamento/economia/DDD424.pdf Agradezco los comentarios de dos árbitros anónimos, los cuales han sido muy útiles para redactar la versión final del trabajo. Los errores que subsistan son, como es natural, míos.

** Profesora principal del Departamento de Economía de la Pontificia Universidad Católica del Perú.
\end{abstract}


to a transition toward another occupation, although in the case of paid house-workers it is usually toward independent labor. Finally, transitions from paid housework to other occupations or inactivity depend on the economic cycle, although the marginal effects are lower for them than for women in general.

Keywords: Labor market, paid house-work, labor transitions, labor supply, gender.

JEL Codes: J15, J16, J21, J46

\section{INTRODUCCIÓN}

El trabajo del hogar remunerado es considerado una ocupación de bajos ingresos y precarias condiciones laborales. Las trabajadoras del hogar no solamente tienen un ingreso total menor que el salario mínimo, sino que sus jornadas laborales son mayores que la jornada legal en el Perú. Asimismo, existe un porcentaje importante de trabajadoras del hogar menores de edad, cuyas jornadas laborales son tan largas como las de las trabajadoras adultas, a pesar de que legalmente sus deberían ser menores. Finalmente, pocas tienen la posibilidad de estudiar, lo que reduce su movilidad laboral e impide una salida hacia ocupaciones de mejores ingresos y condiciones laborales (Organización Internacional del Trabajo - OIT, 2013; Garavito, 2013, 2015). En este contexto es importante analizar la dinámica de este mercado para determinar las probabilidades de salida de esta ocupación, y en qué casos la salida es hacia otra ocupación y no hacia el desempleo o hacia la inactividad.

Entendemos por trabajadoras del hogar a aquellas que realizan las labores diarias de mantenimiento del hogar, excluyendo a aquellos trabajadores que llevan a cabo servicios específicos como jardinería y conducción de vehículos, y a los trabajadores que se ocupan exclusivamente del cuidado especializado de nińos, personas enfermas y adultos mayores (ver OIT, 2013). Si bien muchas trabajadoras del hogar se ocupan también de tareas de cuidado no especializado, el formato de los datos de los paneles de la Encuesta Nacional de Hogares (ENAHO) no permite saber qué tareas específicas llevan a cabo, por lo cual no es posible separarlas efectivamente de las profesionales y técnicas que solamente realizan estas tareas. Aun así, sobre todo en el caso de trabajadoras del hogar que viven en los hogares de sus empleadores — trabajadoras "cama adentro»- las actividades principales que esta realizan están relacionadas a la preparación de comidas y limpieza del hogar ${ }^{1}$. Por otro lado, el crecimiento del porcentaje de trabajadoras del hogar que no viven en casa de sus empleadores — trabajadoras "cama afuera»— ha llevado a cierta especialización en las tareas del hogar demandadas y a relaciones laborales con uno o con varios empleadores a la vez.

\footnotetext{
1 Llamamos trabajadoras «todo servicio» a aquellas que realizan varias tareas en el hogar. Estas trabajadoras son, en su mayoría, trabajadoras «cama adentro».
} 
El objetivo de este trabajo es analizar las transiciones desde el trabajo del hogar remunerado hacia otras ocupaciones, el desempleo y la inactividad, así como los determinantes de estas transiciones. Para ello empleamos los datos panel de la ENAHO para los periodos 1998-2002, 2002-2006 y 2007-20112.

En cuanto a las transiciones entre el trabajo del hogar, otras ocupaciones, el desempleo y la inactividad, postulamos que el aumento en el nivel de educación es un elemento importante en la movilidad laboral. Asimismo, el ciclo económico afecta esta movilidad — si bien las características de este tipo de trabajo pueden alterar dicho efecto. Aun así, el crecimiento del producto está asociado al crecimiento de los ingresos de los hogares demandantes de esta mano de obra, así como a nuevas oportunidades de empleo para las trabajadoras del hogar.

En la segunda sección revisamos trabajos teóricos y empíricos sobre el tema, con énfasis en las transiciones entre el trabajo del hogar y otras ocupaciones. En la tercera sección presentamos los hechos estilizados sobre las transiciones laborales para los periodos mencionados. En la cuarta sección presentamos los estimados de los determinantes de dichas transiciones. Finalmente, en la última sección presentamos las conclusiones de este trabajo.

\section{ESTADO DE LA CUESTIÓN}

Si bien no hemos encontrado análisis teóricos o empíricos de las transiciones entre el trabajo del hogar remunerado (THR) y otras ocupaciones, existen varios trabajos que analizan la movilidad laboral en el Perú en general. Comenzamos entonces por analizar qué factores determinan las transiciones laborales, para luego discutir estos factores para las trabajadoras de hogar.

En general, los cambios en la situación laboral de un trabajador dependen de cambios en las condiciones de demanda y de oferta al mercado de trabajo, así como del contexto macroeconómico y de la estructura institucional ${ }^{3}$. Las condiciones de oferta están asociadas tanto a variables de capital humano, como a características personales y de la familia del trabajador; mientras que las condiciones de demanda están asociadas a la rentabilidad que las empresas esperan tener, dados los precios del bien que venden y los costos de los insumos — factores de producción y materias primas— que demandan.

\footnotetext{
2 Ver el Anexo 1 para una descripción de las bases de datos empleadas.

3 Vamos a mencionar las particularidades de la estructura institucional del trabajo del hogar remunerado en forma transversal a la explicación de los condicionamientos de los efectos de oferta, demanda y contexto económico sobre las transiciones laborales. Para una explicación más detallada de la institucionalidad del THR ver Garavito (2015, 2017). El texto de 2017 ha sido aceptado para su publicación en la revista Cuadernos de Economía de la Universidad Nacional de Colombia. Una versión previa puede verse aquí: http://files.pucp.edu.pe/departamento/economia/DDD420.pdf
} 
En el caso de la trabajadora del hogar, su oferta depende en gran medida de la situación familiar, ya que son los bajos ingresos familiares los que le impiden acceder a niveles de educación que les permitirían trabajar en otras ocupaciones con mayores ingresos y mejores condiciones laborales. Es necesario asimismo tomar en cuenta que esta oferta se da bajo dos modalidades, "cama adentro» y «cama afuera». En el primer caso la trabajadora vive dentro del hogar que demanda sus servicios, lo cual implica más horas de trabajo semanales que en la otra modalidad, así como falta de privacidad, dentro de una relación que no puede considerarse asalariada en el sentido convencional del término. En el segundo caso la trabajadora ofrece sus servicios a uno o varios hogares de manera independiente, lo cual le da cierto control sobre su jornada laboral y sobre sus condiciones de trabajo.

Debemos tomar en cuenta, además, que en el caso del THR quienes demandan esta fuerza laboral no son empresas sino hogares. Así, estos contratan un servicio personal cuyos beneficios y costos son tanto directos como indirectos, y donde el criterio de rentabilidad no se aplica de la misma manera que en las empresas. Los hogares demandan THR para cubrir las horas de trabajo en el hogar que sus propios miembros no están en condiciones o en disposición de cubrir. A esto debemos agregarle una particularidad del vínculo laboral, la cercanía con la familia, lo cual desarrolla vínculos adicionales a los económicos, no siempre en favor de la trabajadora.

Finalmente, el contexto económico puede determinar cambios voluntarios o involuntarios en la situación laboral de un trabajador. En el caso de las salidas involuntarias, son los cambios en el stock de capital de las unidades económicas, así como los cambios en el ciclo los que determinarán la permanencia o salida de un trabajador de un empleo ${ }^{4}$. En el caso de salidas voluntarias, el trabajador lo haría para buscar una ocupación con una mayor remuneración y/o mejores condiciones de trabajo 5 . En relación a este tema, Jovanovic (1984) combina el modelo de búsqueda de Burdett con su propio modelo de emparejamiento y construye uno nuevo donde las transiciones laborales son endógenas. Así, el trabajador cambiará de trabajo, aun si al hacerlo recibiera un salario menor, siempre que haya posibilidades que su salario crezca en el tiempo. Adicionalmente, si el trabajador está desempleado y recibe beneficios laborales, solamente entraría a un nuevo empleo si esperara que la nueva relación laboral sea duradera. Sin embargo, este último caso no se aplica a un país como el Perú, donde no existe un seguro de desempleo ${ }^{6}$.

\footnotetext{
4 Ver Blanchard, Diamond, Hall y Yellen (1989); Mortensen y Pissarides (1994); Lockwood (1991) pasa salidas involuntarias por un shock agregado. o debido a shocks de reasignación de mano de obra entre sectores específicos (Mortensen y Pissarides, 1999; Kletzer, 1998; Lockwood, 1991).

5 Burdett (1978) señala que los trabajadores no acumulan capital humano específico a la firma, por lo cual les es más fácil salir del empleo, mientras Jovanovic (1979) señala que si lo hacen, por lo cual a mayor tiempo en un empleo es menos probable que salgan de este.

6 Si bien existe la Compensación por Tiempo de Servicios (CTS), esta se entrega completa al dejar un empleo formal y vuelve a acumularse al obtener otro empleo.
} 
En el caso que nos ocupa - las transiciones desde el empleo como trabajadoras del hogar a otras ocupaciones - los shocks agregados podrían afectar la demanda de este tipo de trabajo al reducir los ingresos de los empleadores. Sin embargo, en la ENAHO solamente podemos identificar a los empleadores en el caso de las trabajadoras del hogar "cama adentro", que son un porcentaje reducido de la muestra. En cuanto a la salida voluntaria, las trabajadoras del hogar acumulan básicamente capital humano específico a su labor, y solamente podrían acumular otro tipo de capital humano si tuvieran la posibilidad de estudiar, lo cual usualmente no es el caso. En Garavito (2015) vemos que ni siquiera en el caso de las trabajadoras del hogar adolescentes el porcentaje de asistencia a un centro de estudios es mayor al 50\% del total.

Como ya dijimos, no hemos encontrado trabajos específicos sobre transiciones laborales con relación al trabajo del hogar remunerado para el caso del Perú. Sin embargo, algunas de las conclusiones de los trabajos sobre transiciones en general son un insumo para nuestra investigación. En primer lugar están los trabajos sobre vulnerabilidad laboral, entendida esta como una reducción del bienestar del trabajador. Así, Morales, Rodríguez, Higa y Montes (2010), encuentran con datos de las ENAHO que la probabilidad de perder un empleo formal y pasar a uno informal, al desempleo o a la inactividad no se ha reducido entre los ańos 1998 y 2008. Un punto muy importante con relación a nuestro trabajo es no tanto el pase al empleo informal desde uno formal, sino la comprobación que una vez en el sector informal es muy difícil salir de él. Si tomamos en cuenta que el trabajo del hogar remunerado es un empleo básicamente informal, esto nos dice que es muy difícil salir de este trabajo hacia otras ocupaciones. En otro trabajo, Garavito (2010) encuentra — con datos del panel 2004-2006 de la ENAHO para Lima Metropolitana y el resto urbano- que ser mujer, o de etnicidad indígena, aumenta la probabilidad de perder el empleo y de pasar a la inactividad. También encuentra que un mayor nivel de educación aumenta la probabilidad de mantener el empleo en un periodo donde no hay crisis económica. Por otro lado, en el trabajo de Rodríguez y Rodríguez (2012) con los datos de la ENAHO panel 2007-2010, vemos que una mayor educación aumenta la movilidad laboral. Dados los cambios en la estructura del mercado, así como el periodo y áreas de análisis, vemos que la movilidad es mayor entre los más educados y los más jóvenes, y que esta es mayor en el sector urbano que en el sector rural. De nuevo, siendo las trabajadoras del hogar en su mayoría mujeres que viven en los sectores urbanos, deberíamos esperar una mayor movilidad hacia otros empleos ante un aumento de su nivel de educación.

Por lo tanto, postulamos que las salidas hacia otras ocupaciones dependerán de la posibilidad de acumular capital humano no específico al trabajo del hogar remunerado, y de los cambios en el ciclo económico, tomando en cuenta las características específicas de este vínculo laboral. 


\section{TRANSICIONES LABORALES DE LAS TRABAJADORAS DEL HOGAR REMUNERADAS}

En esta sección presentamos los hechos estilizados con respecto a las transiciones entre el trabajo del hogar remunerado, otras ocupaciones, el desempleo y la inactividad con los datos de las ENAHO panel para los periodos 1998-2002, 2002-2006 y 2007-2011. Vamos a analizar tanto los paneles entre los extremos, como los paneles parciales que se construyen a medida que se añade un año más al año inicial ${ }^{7}$.

En Garavito (2015) encontramos que la mayor parte de los trabajadores domésticos, alrededor del $95 \%$ o más, son mujeres. Asimismo, vemos que la edad promedio de las trabajadoras del hogar aumentó entre 1997 y 2013, sobre todo en Lima Metropolitana y en el resto urbano. Esto vino aparejado con un incremento en el porcentaje de trabajadoras del hogar casadas o convivientes, así como una caída en el porcentaje de trabajadoras del hogar bajo la modalidad cama adentro. Adicionalmente, el porcentaje de aquellas trabajadoras que solamente tienen hasta un nivel secundario de educación se ha reducido, lo cual estaría asociado a un recambio generacional - las mujeres más jóvenes no permanecen mucho tiempo en esta ocupación (Garavito, 2017) ${ }^{8}$. Estas tendencias en los datos de corte transversal se reflejan también en los datos de los paneles empleados.

Analizando las transiciones laborales, en los Cuadros 1a-1d podemos ver que la mayoría de trabajadoras del hogar remuneradas se mantiene en su ocupación en los periodos analizados, siendo el mayor porcentaje para el panel 1998-2000 (38,5\%) y el menor para el panel 1998-2001 (13,0\%). Vemos asimismo que en todos los casos las trabajadoras que dejan el THR pasan en su mayoría a la inactividad y en segundo lugar a otras ocupaciones. En el Anexo 2 podemos ver para los tres paneles que las trabajadoras del hogar que pasan a otras ocupaciones tienen un mayor nivel de educación y son en la mayor parte de los casos más jóvenes que aquellas que se quedan en el THR. Asimismo, en general las trabajadoras que dejan el THR y pasan a la inactividad tienen una mayor edad promedio. Por otro lado, en el Anexo 3 vemos que la permanencia en el THR es mayor en el área urbana que en el área rural, y que la movilidad hacia otras ocupaciones también es mayor, lo cual reflejaría las oportunidades laborales en las ciudades. Es también evidente que el número de trabajadoras del hogar en el sector rural es muy reducido en relación con el sector urbano.

En los Cuadros 2a-2d vemos de nuevo que la mayoría de trabajadoras del hogar remuneradas se mantiene en su ocupación en los periodos analizados, siendo el mayor porcentaje para el panel 2002-2003 (47,5\%) y el menor para el panel 2002-2004 $(37,1 \%)$. Tanto la permanencia entre los dos primeros ańos de este panel, como entre los extremos, es mayor que los porcentajes respectivos en el panel anterior, lo cual muestra una menor movilidad de las trabajadoras del hogar. Asimismo, en los paneles 2004-2006

\footnotetext{
Trabajamos con los datos expandidos. Sobre esto ver Deaton (1997).

8 Sobre la fecha de publicación ver nota 3 .
} 
y 2007-2011 las trabajadoras del hogar que hablan una lengua nativa tienen una menor probabilidad de salir del THR hacia otra ocupación.

En cuanto a las trabajadoras del hogar que dejan esta ocupación, vemos que la mayoría pasa en primer lugar a otra ocupación y en segundo lugar a la inactividad. Esto revela un cambio en los patrones de las transiciones hacia fuera del THR, que estaría asociada al mayor crecimiento del Producto Interno Bruto (PIB) real entre 2002-2006 con relación al periodo 1998 y 2002. En el Anexo 4 podemos ver que luego de un crecimiento moderado entre 1998 y 2000, el producto cae para luego recuperarse, creciendo a tasas anuales de alrededor del $10 \%$ hasta 2004, lo cual configura un escenario de crecimiento durante el periodo del segundo panel, con el consiguiente aumento de los ingresos. Esto explicaría la reducción de la movilidad hacia afuera de otras trabajadoras del hogar debido a la mayor demanda por sus servicios; mientras la aparición de nuevas oportunidades de empleo derivada del crecimiento del producto explicaría la mayor salida de las trabajadoras hacia otras ocupaciones que a la inactividad, al contrario que en panel anterior.

Finalmente, en los Cuadros 3a-3d, podemos ver que en el panel 2007-2008 el porcentaje de trabajadoras del hogar que mantiene la misma ocupación es de 50,0\%, bajando a 45,2\% en el panel 2007-2009, justo antes de la crisis global del año 2009. Luego de esta, los porcentajes son de 37,9\% y 22,3\% para los paneles 2007-2010 y 2007-2011, respectivamente. La caída en el porcentaje de trabajadoras del hogar que se mantienen en su ocupación en el panel completo se debería en parte a la caída en el número de casos del panel al agregar el año 2011, así como al cambio en la metodología para medir la pobreza en la ENAHO.

Vemos asimismo que la principal salida del THR es hacia otras ocupaciones y no a la inactividad, y que este porcentaje aumenta luego de la crisis de 2009. En este caso se trata no de solamente una menor demanda debido a la caída del producto, sino también de una salida hacia ocupaciones que no necesariamente llevan a mayores ingresos. Para examinar este punto debemos analizar a qué ocupaciones específicas salen las trabajadoras del hogar en los tres paneles.

Cuadro 1a. Perú Total, Panel 1998-1999: transiciones laborales (\%)

\begin{tabular}{l|l|c|c|c|c|c|r}
\hline \multicolumn{2}{c|}{} & \multicolumn{7}{c}{1999} \\
\cline { 3 - 8 } \multicolumn{2}{c|}{} & Trab. del hogar & Otras ocup. & Desemp. & Inactivos & Total & Población \\
\hline \multirow{4}{*}{1998} & Trabajador del hogar & 24,8 & 18,1 & 0,0 & 57,1 & 100,0 & 22502 \\
& Otras ocupaciones & 0,0 & 86,4 & 1,3 & 12,3 & 100,0 & 583119 \\
& Desempleados & 0,0 & 74,9 & 9,4 & 15,7 & 100,0 & 26995 \\
& Inactivos & 1,2 & 26,1 & 3,8 & 68,9 & 100,0 & 325663 \\
\cline { 2 - 8 } & Población & 9523 & 613158 & 22689 & 312909 & 100,0 & 958279 \\
\hline
\end{tabular}

Fuente: ENAHO-INEI Panel 1998-1999. 
Cuadro 1b. Perú Total, Panel 1998-2000: transiciones laborales (\%)

\begin{tabular}{l|l|c|c|c|c|c|r}
\hline \multicolumn{2}{c|}{} & \multicolumn{7}{c}{2000} \\
\cline { 3 - 8 } \multicolumn{2}{c|}{} & Trab. del hogar & Otras ocup. & Desemp. & Inactivos & Total & Población \\
\hline \multirow{3}{*}{1998} & Trabajador del hogar & 38,5 & 18,2 & 0,0 & 43,3 & 100,0 & 22502 \\
& Otras ocupaciones & 0,6 & 83,1 & 2,8 & 13,5 & 100,0 & 603118 \\
& Desempleados & 0,0 & 55,1 & 25,0 & 19,9 & 100,0 & 34804 \\
& Inactivos & 3,9 & 31,4 & 6,4 & 58,3 & 100,0 & 340373 \\
\cline { 2 - 8 } & Población & 25246 & 631426 & 47794 & 296331 & 100,0 & 1000797 \\
\hline
\end{tabular}

Fuente: ENAHO-INEI Panel 1998-2000.

Cuadro 1c. Perú Total, Panel 1998-2001: transiciones laborales (\%)

\begin{tabular}{l|c|c|c|c|c|c|r}
\hline \multicolumn{2}{c|}{} & \multicolumn{7}{c}{2001} \\
\cline { 3 - 8 } \multicolumn{2}{c|}{} & Trab. del hogar & Otras ocup. & Desemp. & Inactivos & Total & Población \\
\hline \multirow{4}{*}{1998} & Trabajador del hogar & 13,0 & 11,4 & 0,0 & 75,6 & 100,0 & 22502 \\
& Otras ocupaciones & 1,2 & 82,4 & 2,4 & 14,0 & 100,0 & 590770 \\
& Desempleados & 0,0 & 58,8 & 14,7 & 26,5 & 100,0 & 33405 \\
& Inactivos & 1,4 & 33,5 & 3,0 & 62,1 & 100,0 & 325144 \\
\cline { 2 - 8 } & Población & 14712 & 617973 & 28375 & 310761 & 100,0 & 971821 \\
\hline
\end{tabular}

Fuente: ENAHO-INEI Panel 1998-2001.

Cuadro 1d. Perú Total, Panel 1998-2002: transiciones laborales (\%)

\begin{tabular}{l|c|c|c|c|c|c|r}
\hline \multicolumn{2}{c|}{} & \multicolumn{6}{c}{2002} \\
\cline { 3 - 8 } \multicolumn{2}{c|}{} & Trab. del hogar & Otras ocup. & Desemp. & Inactivos & Total & Población \\
\hline \multirow{4}{*}{1998} & Trabajador del hogar & 25,4 & 24,7 & 1,0 & 48,9 & 100,0 & 22502 \\
& Otras ocupaciones & 0,4 & 80,6 & 2,1 & 16,9 & 100,0 & 559067 \\
& Desempleados & 3,9 & 51,5 & 1,8 & 42,8 & 100,0 & 32959 \\
& Inactivos & 1,8 & 38,3 & 3,3 & 56,6 & 100,0 & 307141 \\
\cline { 2 - 8 } & Población & 14702 & 590799 & 22891 & 293507 & 100,0 & 921669 \\
\hline
\end{tabular}

Fuente: ENAHO-INEI Panel 1998-2002.

Cuadro 2a. Perú Total, Panel 2002-2003 transiciones laborales (\%)

\begin{tabular}{l|c|c|c|c|c|c|r}
\hline \multicolumn{2}{c|}{} & \multicolumn{7}{c}{2003} \\
\cline { 3 - 8 } \multicolumn{2}{c|}{} & Trab. del hogar & Otras ocup. & Desemp. & Inactivos & Total & Población \\
\hline \multirow{3}{*}{2002} & Trabajador del hogar & 47,5 & 33,3 & 1,9 & 17,3 & 100,0 & 189832 \\
& Otras ocupaciones & 0,7 & 87,6 & 2,3 & 9,4 & 100,0 & 8089057 \\
& Desempleados & 0,4 & 52,0 & 15,1 & 32,5 & 100,0 & 367618 \\
& Inactivos & 1,2 & 30,0 & 4,7 & 64,1 & 100,0 & 4018723 \\
\cline { 2 - 8 } & Población & 193671 & 8547329 & 431753 & 2576637 & 100,0 & 12665230 \\
\hline
\end{tabular}

Fuente: ENAHO-INEI Panel 2002-2003. 
Cuadro 2b. Perú Total, Panel 2002-2004 transiciones laborales (\%)

\begin{tabular}{l|c|c|c|c|c|c|r}
\hline \multicolumn{2}{c|}{} & \multicolumn{7}{c}{2004} \\
\cline { 3 - 8 } \multicolumn{2}{c|}{} & Trab. del hogar & Otras ocup. & Desemp. & Inactivos & Total & Población \\
\hline \multirow{3}{*}{2002} & Trabajador del hogar & 37,1 & 36,8 & 3,1 & 23,0 & 100,0 & 284706 \\
& Otras ocupaciones & 0,6 & 87,2 & 1,8 & 10,4 & 100,0 & 11101346 \\
& Desempleados & 1,9 & 57,6 & 10,3 & 30,2 & 100,0 & 551082 \\
& Inactivos & 1,8 & 35,7 & 5,4 & 57,1 & 100,0 & 5493347 \\
\cline { 2 - 8 } & Población & 278778 & 12063952 & 561349 & 4525402 & 100,0 & 17429481 \\
\hline
\end{tabular}

Fuente: ENAHO-INEI Panel 2002-2004.

Cuadro 2c. Perú Total, Panel 2002-2005 transiciones laborales (\%)

\begin{tabular}{l|c|c|c|c|c|c|r}
\hline \multicolumn{2}{c|}{} & \multicolumn{7}{c}{2005} \\
\cline { 3 - 8 } \multicolumn{2}{c|}{} & Trab. del hogar & Otras ocup. & Desemp. & Inactivos & Total & Población \\
\hline \multirow{3}{*}{2002} & Trabajador del hogar & 41,9 & 31,3 & 1,2 & 25,6 & 100,0 & 219500 \\
& Otras ocupaciones & 0,8 & 85,6 & 2,0 & 11,6 & 100,0 & 9774991 \\
& Desempleados & 1,9 & 55,1 & 12,6 & 30,4 & 100,0 & 464729 \\
& Inactivos & 1,7 & 39,6 & 4,5 & 54,2 & 100,0 & 4814803 \\
\cline { 2 - 8 } & Población & 257948 & 10602362 & 471596 & 3942117 & 100,0 & 15274023 \\
\hline
\end{tabular}

Fuente: ENAHO-INEI Panel 2002-2005.

Cuadro 2d. Perú Total, Panel 2002-2006 transiciones laborales (\%)

\begin{tabular}{l|l|c|c|c|c|c|r}
\hline \multicolumn{2}{c|}{} & \multicolumn{7}{c}{2006} \\
\cline { 2 - 8 } \multicolumn{2}{c|}{} & Trab. del hogar & Otras ocup. & Desemp. & Inactivos & Total & Población \\
\hline \multirow{3}{*}{2002} & Trabajador del hogar & 39,1 & 33,1 & 3,4 & 24,4 & 100,0 & 227290 \\
& Otras ocupaciones & 0,7 & 86,8 & 1,4 & 11,1 & 100,0 & 9438776 \\
& Desempleados & 2,0 & 56,2 & 8,6 & 33,2 & 100,0 & 444972 \\
& Inactivos & 2,7 & 41,0 & 4,8 & 51,5 & 100,0 & 4601976 \\
\cline { 2 - 8 } & Población & 292968 & 10408171 & 394078 & 3617797 & 100,0 & 14713014 \\
\hline
\end{tabular}

Fuente: ENAHO-INEI Panel 2002-2006.

Cuadro 3a. Perú Total, Panel 2007-2008 transiciones laborales (\%)

\begin{tabular}{l|l|c|c|c|c|c|r}
\hline \multicolumn{2}{c|}{} & \multicolumn{7}{c}{2008} \\
\cline { 3 - 8 } \multicolumn{2}{c|}{} & Trab. del hogar & Otras ocup. & Desemp. & Inactivos & Total & Población \\
\hline \multirow{3}{*}{2007} & Trabajador del hogar & 50,0 & 29,1 & 1,5 & 19,4 & 100,0 & 554117 \\
& Otras ocupaciones & 0,9 & 86,0 & 2,2 & 10,9 & 100,0 & 17158622 \\
& Desempleados & 3,1 & 45,1 & 13,8 & 38,0 & 100,0 & 780806 \\
& Inactivos & 2,0 & 27,6 & 4,1 & 66,3 & 100,0 & 7286075 \\
\cline { 2 - 8 } & Población & 600463 & 17277242 & 792993 & 7108921 & 100,0 & 25779620 \\
\hline
\end{tabular}

Fuente: ENAHO-INEI Panel 2007-2008. 
Cuadro 3b. Perú Total, Panel 2007-2009 transiciones laborales (\%)

\begin{tabular}{l|l|c|c|c|c|c|r}
\hline \multicolumn{2}{c|}{} & \multicolumn{7}{c}{2009} \\
\cline { 3 - 8 } \multicolumn{2}{c|}{} & Trab. del hogar & Otras ocup. & Desemp. & Inactivos & Total & Población \\
\hline \multirow{3}{*}{2007} & Trabajador del hogar & 45,2 & 37,3 & 3,5 & 14,0 & 100,0 & 482649 \\
& Otras ocupaciones & 0,8 & 85,9 & 1,9 & 11,4 & 100,0 & 15354040 \\
& Desempleados & 0,6 & 52,6 & 9,5 & 37,3 & 100,0 & 667009 \\
& Inactivos & 2,0 & 31,5 & 4,4 & 62,1 & 100,0 & 6393448 \\
\cline { 2 - 8 } & Población & 466677 & 15748993 & 651643 & 6029833 & 100,0 & 22897146 \\
\hline
\end{tabular}

Fuente: ENAHO-INEI Panel 2007-2009.

Cuadro 3c. Perú Total, Panel 2007-2010 transiciones laborales (\%)

\begin{tabular}{l|l|c|c|c|c|c|r}
\hline \multicolumn{2}{c|}{} & \multicolumn{7}{c}{2010} \\
\cline { 3 - 8 } \multicolumn{2}{c|}{} & Trab. del hogar & Otras ocup. & Desemp. & Inactivos & Total & Población \\
\hline \multirow{3}{*}{2007} & Trabajador del hogar & 37,9 & 46,4 & 2,5 & 13,2 & 100,0 & 436595 \\
& Otras ocupaciones & 0,8 & 84,9 & 1,5 & 12,8 & 100,0 & 16514834 \\
& Desempleados & 0,5 & 60,2 & 10,4 & 28,9 & 100,0 & 691946 \\
& Inactivos & 2,4 & 32,3 & 3,0 & 62,3 & 100,0 & 6347893 \\
\cline { 2 - 8 } & Población & 448776 & 16688861 & 527922 & 6325709 & 100,0 & 23991268 \\
\hline
\end{tabular}

Fuente: ENAHO-INEI Panel 2007-2010.

Cuadro 3d. Perú Total, Panel 2007-2011 transiciones laborales (\%)

\begin{tabular}{l|l|c|c|c|c|c|r}
\hline \multicolumn{2}{c|}{} & \multicolumn{7}{c}{2011} \\
\cline { 3 - 8 } \multicolumn{2}{c|}{} & Trab. del hogar & Otras ocup. & Desemp. & Inactivos & Total & Población \\
\hline \multirow{3}{*}{2007} & Trabajador del hogar & 22,4 & 57,5 & 1,9 & 18,2 & 100,0 & 404010 \\
& Otras ocupaciones & 0,8 & 86,7 & 1,3 & 11,2 & 100,0 & 17321105 \\
& Desempleados & 0,0 & 66,6 & 4,2 & 29,2 & 100,0 & 781242 \\
& Inactivos & 3,1 & 37,5 & 6,0 & 53,4 & 100,0 & 6825524 \\
\cline { 2 - 8 } & Población & 446722 & 18328831 & 676199 & 5880128 & 100,0 & 25331881 \\
\hline
\end{tabular}

Fuente: ENAHO-INEI Panel 2007-2011.

En los Cuadros 4a-4c presentamos los datos de las transiciones entre ocupaciones para los tres paneles ${ }^{9}$, lo cual nos permite comprender mejor la dinámica de estos cambios. En estos cuadros ya no trabajamos con la población en edad de trabajar (PET), sino con la población económicamente activa (PEA) ocupada.

Vemos en primer lugar que el $45,7 \%$ de trabajadoras del hogar remuneradas no cambia de ocupación en el periodo 1998-2002, el 54,3\% en el periodo 2002-2006 y solamente el 29,9\% en el periodo 2007-2011. Es decir, la movilidad laboral fuera del THR disminuye en el panel en el cual no hay crisis económica, para luego aumentar en el tercer panel. En segundo lugar, vemos que para el caso panel 1998-2002, el 22,7\% de

9 Las transiciones parciales entre ocupaciones se pueden ver en los Anexos 5, 6 y 7. 
las trabajadoras del hogar remuneradas pasan a ser empleadas, mientras que el 21,6\% pasan a ser trabajadoras independientes ${ }^{10}$. En el panel 2002-2006, vemos que el 13,0\% pasan a ser obreras y el $10,1 \%$ a ser trabajadoras independientes. Finalmente, en el periodo 2007-2011, el 26,1\% pasan a ser trabajadoras independientes y el 22,70\% a ser obreras. Una tendencia constante es que el porcentaje de trabajadoras del hogar que pasan a ser TFNR se reduce en el periodo cubierto por los tres paneles. Esto se debería a la mayor salida de las trabajadoras del hogar remuneradas hacia otras ocupaciones.

En la siguiente sección, llevamos a cabo un análisis de los determinantes de las transiciones en el tiempo desde el trabajo del hogar remunerado hacia otras ocupaciones, el desempleo o la inactividad para todos los paneles.

Cuadro 4a. Panel 1998-2002: transiciones entre ocupaciones

\begin{tabular}{|c|c|c|c|c|c|c|c|c|}
\hline & \multicolumn{7}{|c|}{2002} \\
\hline & & $\begin{array}{c}\text { Emplea- } \\
\text { dor }\end{array}$ & $\begin{array}{l}\text { Indepen- } \\
\text { diente }\end{array}$ & Empleado & Obrero & TFNR & $\begin{array}{l}\text { Trab. } \\
\text { hogar }\end{array}$ & Población \\
\hline \multirow{7}{*}{1998} & Empleador & 28,6 & 27,6 & 21,7 & 18,5 & 3,6 & 0,0 & 40160 \\
\hline & Trab. independiente & 8,4 & 74,3 & 5,8 & 7,9 & 2,5 & 1,1 & 182912 \\
\hline & Empleado & 0,0 & 25,3 & 65,4 & 8,0 & 1,3 & 0,0 & 67252 \\
\hline & Obrero & 1,9 & $15,4^{*}$ & 9,8 & 63,8 & 9,1 & 0,0 & 114 \\
\hline & TFNR & 1,1 & 15,8 & 5,0 & 17,7 & 56,6 & 3,8 & 79127 \\
\hline & Trabajador del hogar & 0,0 & 21,6 & 22,7 & 0,0 & 10,0 & 45,7 & 12524 \\
\hline & Población & 29850 & $196827^{*}$ & 81275 & 113934 & 63331 & 10770 & 495987 \\
\hline
\end{tabular}

Fuente: ENAHO-INEI Panel 1998-2002.

* El rubro «otros» de trabajos no especificados ha sido incluido en la categoría «independiente«.

Cuadro 4b. Panel 2002-2006: transiciones entre ocupaciones

\begin{tabular}{|c|c|c|c|c|c|c|c|c|}
\hline & \multicolumn{7}{|c|}{2006} \\
\hline & & $\begin{array}{c}\text { Emplea- } \\
\text { dor }\end{array}$ & $\begin{array}{l}\text { Indepen- } \\
\text { diente }\end{array}$ & Empleado & Obrero & TFNR & $\begin{array}{l}\text { Trab. } \\
\text { hogar }\end{array}$ & Población \\
\hline \multirow{7}{*}{2002} & Empleador & 42,8 & $41,9^{*}$ & 4,4 & 8,2 & 2,6 & 0,1 & 540884 \\
\hline & Trab. independiente & 8,4 & $70,5^{*}$ & 3,9 & 8,7 & 8,7 & 7,8 & $3538645^{*}$ \\
\hline & Empleado & 1,3 & $12,9^{*}$ & 72,5 & 9,0 & 3,1 & 1,1 & 1435677 \\
\hline & Obrero & 4,3 & $23,3^{*}$ & 11,0 & 53,3 & 6,7 & 1,4 & 1427329 \\
\hline & TFNR & 0,8 & $20,3^{*}$ & 4,0 & 13,6 & 60,2 & 1,1 & 1811147 \\
\hline & Trabajador del hogar & 5,5 & 10,1 & 7,2 & 13,0 & 9,9 & 54,3 & 169887 \\
\hline & Población & 586641 & $3785658^{*}$ & 1438074 & 1425832 & 1676784 & 196957 & 8923569 \\
\hline
\end{tabular}

Fuente: ENAHO-INEI Panel 2002-2006.

* El rubro «otros» de trabajos no especificados ha sido incluido en la categoría «independiente«.

10 Esto es un cambio con respecto a los resultados de estudios de la década de 1970, citados en Bunster, y Chaney (1985), donde se señalaba que la mayoría de trabajadoras del hogar pasaba a trabajar como vendedoras ambulantes (trabajadoras independientes), pero casi nunca a ocupaciones «white collar» como empleadas. 
Cuadro 4c. Panel 2007-2011: transiciones entre ocupaciones

\begin{tabular}{l|c|c|c|c|c|c|c|c}
\hline \multirow{2}{*}{} & \multicolumn{9}{|c}{2011} \\
\cline { 3 - 9 } \multicolumn{2}{c|}{} & Empleador & $\begin{array}{c}\text { Indepen- } \\
\text { diente }\end{array}$ & Empleado & Obrero & TFNR & $\begin{array}{c}\text { Trab. } \\
\text { hogar }\end{array}$ & Población \\
\hline \multirow{6}{*}{2007} & Empleador & 35,7 & 38,5 & 7,7 & 10,9 & 6,7 & 0,5 & 1088454 \\
& Trab. independiente & 6,0 & $71,3^{*}$ & 6,6 & 8,5 & 6,5 & 1,1 & 5953831 \\
& Empleado & 3,2 & 9,2 & 75,2 & 9,4 & 2,1 & 0,9 & 3366529 \\
& Obrero & 3,7 & $28,1^{*}$ & 12,4 & 51,9 & 3,6 & 0,3 & 2860693 \\
& TFNR & 3,9 & $20,4^{*}$ & 8,5 & 9,3 & 56,0 & 1,9 & 2711826 \\
& Trabajador del hogar & 0,0 & $26,1^{*}$ & 21,3 & 22,7 & 0,0 & 29,9 & 337434 \\
\cline { 2 - 10 } & Población & 1064478 & $6422729^{*}$ & 3656568 & 2755534 & 2154692 & 264766 & 16318767 \\
\hline
\end{tabular}

Fuente: ENAHO-INEI Panel 2007-2011.

* El rubro «otros» de trabajos no especificados ha sido incluido en la categoría «independiente»».

\section{ESTIMACIONES DE LOS DETERMINANTES DE LAS TRANSICIONES LABORALES}

En esta sección presentamos las estimaciones de los determinantes de las transiciones desde el trabajo del hogar remunerado hacia otras ocupaciones, y hacia el desempleo o la inactividad ${ }^{11}$. De acuerdo a esto planteamos las siguientes categorías para la variable endógena (tran):
tran $=1 \quad$ Trabajadora del hogar $\rightarrow$ Trabajadora del hogar
tran $=2 \quad$ Trabajadora del hogar $\rightarrow$ Otra ocupación
tran $=3 \quad$ Trabajadora del hogar $\rightarrow$ Desempleo o inactividad

Las variables exógenas serán las siguientes:

- Nivel de educación: variable que tiene once categorías: sin nivel, inicial, primaria incompleta, primaria completa, secundaria incompleta, secundaria completa, superior no universitaria incompleta, superior no universitaria completa, superior universitaria incompleta, superior universitaria completa y posgrado. Esperamos que un aumento del nivel de educación esté asociado positivamente al paso a otra ocupación con relación a permanecer en el THR.

- Edad: variable numérica ligada tanto a la mayor experiencia en las tareas realizadas, como a los cambios en las oportunidades laborales al aumentar la edad. Esperamos que a mayor edad sea menos probable la salida del THR, dado que las otras oportunidades laborales se reducirían. En cuanto a su efecto sobre la salida hacia la inactividad, hay dos efectos, el hecho que a mayor edad hay una menor

\footnotetext{
11 Juntamos estas dos opciones por dos razones. La primera que hay muy pocos casos de salida del THR hacia el desempleo; la segunda que los resultados de la salida del THR a la inactividad son muy parecidos a los hallados cuando se juntas las dos opciones.
} 
probabilidad de continuar trabajando, y el hecho que las trabajadoras del hogar son pobres lo cual implicaría una mayor permanencia en el THR.

- Jefe de hogar: variable dicotómica cuyo valor es igual a 1 si la trabajadora es jefa de su hogar y 0 si no lo es. Esperamos que las trabajadoras jefas de hogar busquen salir del THR dados los bajos salarios de esta ocupación; sin embargo su condición de proveedoras del sustento de su hogar llevaría a que se mantengan en dicho empleo.

- Lengua materna nativa: variable dicotómica cuyo valor es igual a 1 si la trabajadora habla quechua, aimara o una lengua nativa y 0 en caso contrario. Esperamos una menor movilidad fuera del THR si la lengua materna es nativa, ya que esto está asociado a menores oportunidades laborales, sobre todo en el sector urbano.

- Año: variables dicotómicas que son iguales a 1 en un año determinado y cero en otro caso. Con estas variables buscamos captar los efectos del ciclo económico sobre la demanda por THR.

Entonces, la ecuación a estimar para las transiciones laborales sería la siguiente:

$$
\text { tran }=\alpha_{0}+\alpha_{1} \text { NivEduca }+\alpha_{2} \text { Edad }+\alpha_{3} \text { JefeHogar }+\alpha_{4} \text { LenguaNativa }+\alpha_{5} \text { Año }+u
$$

En los tres paneles, el Test de Hausman confirma que el panel de efectos fijos es el modelo adecuado. El programa Stata no tiene un comando para estimar ecuaciones Logit Multinomiales en el panel con efectos fijos; y el comando GLLAMM solamente estima ecuaciones Logit Multinomiales en el panel con efectos aleatorios. Por lo tanto, siguiendo a Long y Freese (2014) quienes señalan que los resultados de una estimación Logit Multinomial se pueden aproximar por los resultados de dos estimaciones Logit Binomiales, hicimos las estimaciones respectivas entre las categorías 1 y 2 (tran $=1$ versus tran $=2)$, y 1 y 3 (tran $=1$ versus tran $=3$ ). Los estimados se encuentran en los Cuadros 5a y 5b.

En el Cuadro $5 \mathrm{a}^{12}$ vemos que un aumento del nivel de educación está asociado a que la trabajadora del hogar pase a otra ocupación en los tres paneles, si bien el efecto marginal es mayor en el panel 2002-2006, periodo en el cual no hay crisis económica ${ }^{13}$. Debemos recordar que si bien en los tres paneles las trabajadoras del hogar salen hacia el trabajo independiente, esta es la primera opción solamente para los dos últimos paneles, mientras que es la segunda opción para el panel 1998-2002, donde la primera opción es pasar a ser empleada. Es decir, un mayor nivel de educación lleva a una mayor movilidad laboral ${ }^{14}$, más en el caso de las trabajadoras del hogar no implica necesariamente una mejora en la calidad del empleo. En cuanto a la edad promedio, el efecto sobre la salida a otra ocupación solamente es positivo para el segundo panel (2002-2006)

\footnotetext{
12 Empleamos el panel 2007-2010 porque no fue posible estimar los efectos marginales en el panel 2007-2011.

13 Ver Anexo 4.

${ }^{14}$ Lo cual coincide con lo hallado por Rodríguez y Rodríguez (2012) para el panel 2007-2010.
} 
y para su panel parcial (2004-2006), siendo negativo en todos los otros casos. Es decir, en general a mayor edad la probabilidad de permanecer en el THR es mayor, tal como esperábamos. En el caso de las jefas de hogar, estas tienen una mayor probabilidad de salir hacia otras ocupaciones en el panel 1998-2002 y una menor probabilidad de salida en los paneles restantes. Recordando que solamente es posible identificar a las jefas de hogar en el caso de las trabajadoras "cama afuera», esto implica un mayor efecto de su condición de proveedoras del sustento del hogar sobre su movilidad hacia ocupaciones mejor remuneradas a partir de la década de 2000. En los paneles donde tenemos el dato de la lengua materna (2004-2006 y 2007-2011) vemos que la probabilidad de salir hacia otra ocupación es menor para aquellas trabajadoras cuya lengua materna es nativa, lo cual se explicaría por las menores oportunidades de empleo, sobre todo en las ciudades. Finalmente, en el caso de los años - que representan al ciclo económico- vemos que la probabilidad de salir del THR aumenta en los ańos de crecimiento y se reduce en los años de caída del producto para los dos primeros paneles. Así, la probabilidad de salir hacia otra ocupación cae a partir del año 2001 en el panel 1998-2002, a partir del año 2003 en el panel 2002-2006, a partir del año 2004 en el panel parcial del segundo panel. En el caso del panel 2007-2010, la probabilidad de salir del THR aumenta aún después de la crisis, y la salida hacia otras ocupaciones es mayor en el año luego de la caída del PIB de 2009. Esto estaría relacionado a caídas en la demanda por los servicios de las trabajadoras del hogar, que en su mayoría saldrán hacia el trabajo independiente.

En el Cuadro 5b vemos que un mayor nivel de educación está asociado a una mayor probabilidad de salir hacia el desempleo o la inactividad en todos los casos, siendo mayor el efecto marginal en el panel 2002-2006, en el cual el PIB crece. Esto significa que si bien obtener un mayor nivel de educación lleva a la salida del THR tal como vimos en el Cuadro 5a, no todas las trabajadoras encuentran empleo o pueden iniciar un negocio propio como trabajadoras independientes. En el Anexo 8 podemos ver que esto coincide con estimaciones para las mujeres como un grupo, independientemente de su ocupación. En el caso de la edad, encontramos que a mayor edad es menor la probabilidad de salir del THR hacia la inactividad o hacia el desempleo para todos los paneles, siendo el efecto marginal mayor —en valor absoluto- en el segundo panel. Si comparamos este resultado con las estimaciones del Anexo 8 vemos que a mayor edad la probabilidad de pasar al desempleo o a la inactividad es mayor, salvo para el panel 2007-2011. Vemos asimismo que las trabajadoras que son jefas de hogar tienen una mayor probabilidad de salir hacia el desempleo o la inactividad en el primer panel, y una menor probabilidad en los otros periodos. En los paneles 2004-2006 y 2007-2010 el hablar una lengua materna nativa aumenta la probabilidad de salir del THR hacia el desempleo o la inactividad, lo cual coincide con lo hallado para las mujeres en general en el panel 2007-2010 (ver Anexo 8) ${ }^{15}$.

${ }^{15}$ Asimismo, estos resultados coinciden con Garavito (2010) quien encuentra que las personas que tienen una lengua materna nativa experimentan una mayor probabilidad de perder su empleo y pasar al desempleo o a la inactividad. 
Estos dos últimos resultados son consistentes con lo hallado en el Cuadro 5a sobre la salida a otras ocupaciones. Finalmente, en cuanto al efecto del ciclo económico sobre la salida del THR hacia el desempleo o hacia la inactividad, esta probabilidad aumenta en el panel 1998-2002, en el cual hay crisis económica, y disminuye en el panel 2002-2006, donde el PIB crece. Si comparamos esto con los cuadros para las mujeres en general del Anexo 8 vemos que la probabilidad de pasar a la inactividad disminuye en ambos paneles. La diferencia con respecto al THR en el primer panel podría explicarse por una mayor estabilidad relativa en la ocupación del resto de mujeres trabajadoras, especialmente en el sector formal. En cuanto al panel 2007-2010, la probabilidad de salir del THR hacia el desempleo o la inactividad es aumenta a partir del año 2009, en medio de un contexto donde la salida a la inactividad de las mujeres aumenta en todo el periodo.

Vemos así que las transiciones desde el trabajo del hogar remunerado hacia otras ocupaciones, o hacia la inactividad y el desempleo, están asociadas tanto al cambio en variables individuales, como la educación, la edad, y la lengua materna, como al ciclo económico y a la estructura institucional de esta ocupación. En la siguiente sección presentamos las conclusiones de esta investigación.

Cuadro 5a. Regresiones Logit Binomial en el panel

\begin{tabular}{l|c|c|c|c|c}
\hline Logit binomial en el panel & \multicolumn{5}{|c}{ Pasa a otra ocupación } \\
\hline Base = sigue como THR & $1998-2002$ & $2002-2006$ & $2007-2010$ & $2004-2006$ & $2007-2010$ \\
\hline Nivel de educación & $0,5147^{* * *}$ & $0,3939^{* * *}$ & $0,4849^{* * *}$ & $0,3910^{* * *}$ & $0,4265^{* * *}$ \\
Edad promedio & $-0,1232^{* * *}$ & $0,0072^{* *}$ & $-0,4437^{* * *}$ & $0,2942^{* * *}$ & $-0,3949^{* * *}$ \\
Jefe de hogar & $0,2190^{* * *}$ & $-0,1026$ & $-0,9774^{* * *}$ & $-0,5418^{* * *}$ & $-0,8783^{* * *}$ \\
Lengua materna nativa & $\mathrm{n}, \mathrm{d}$, & $\mathrm{n}, \mathrm{d}$, & $\mathrm{n}, \mathrm{d}$, & $-0,6040^{* * *}$ & $-2,5727^{* * *}$ \\
Año t+1 & $0,3973^{* * *}$ & $0,2789^{* * *}$ & $0,7167^{* * *}$ & $-0,2679^{* * *}$ & $0,7331^{* * *}$ \\
Año t+2 & $0,3865^{* * *}$ & $0,0846^{* * *}$ & $1,3458^{* * *}$ & $-0,5875^{* * *}$ & $1,3355^{* * *}$ \\
Año t+3 & $-0,2007^{* * *}$ & $-0,0453^{* * *}$ & $1,7632^{* * *}$ & & $1,7267^{* * *}$ \\
Año t+4 & $-0,4078^{* * *}$ & $-0,0687^{* * *}$ & & & \\
\hline Efectos marginales & $1998-2002$ & $2002-2006$ & $2007-2010$ & $2004-2006$ & $2007-2010$ \\
\hline Nivel de educación & $0,00002^{* * *}$ & $0,0329^{* * *}$ & $1,82 \mathrm{e}-06^{* * *}$ & $3,58 \mathrm{e}-06^{* * *}$ & $4,43 \mathrm{e}-06^{* * *}$ \\
Edad promedio & $0,00001^{* * *}$ & $0,0006^{* * *}$ & $-1,67 \mathrm{e}-06^{* * *}$ & $2,70 \mathrm{e}-06^{* * *}$ & $-4,10 \mathrm{e}-06^{* * *}$ \\
Jefe de hogar & $-4,90 \mathrm{e}-06^{* * *}$ & $-0,0010$ & $-2,98 \mathrm{e}-06^{* * *}$ & $-5,78 \mathrm{e}-06^{* * *}$ & $-7,54 \mathrm{e}-06^{* * *}$ \\
Lengua materna nativa & $\mathrm{n}, \mathrm{d}$, & $\mathrm{n}, \mathrm{d}$, & $\mathrm{n}, \mathrm{d}$, & $-6,81 \mathrm{e}-06^{* * *}$ & $-0,00001^{* * *}$ \\
Año t+1 & $0,00001^{* * *}$ & $0,0215^{* * *}$ & $3,25 \mathrm{e}-06^{* * *}$ & $-2,56 \mathrm{e}-06^{* * *}$ & $9,23 \mathrm{e}-06^{* * *}$ \\
Año t+2 & $0,00001^{* * *}$ & $0,0069^{* * *}$ & $7,71 \mathrm{e}-06^{* * *}$ & $-6,08 \mathrm{e}-06^{* * *}$ & $0,00002^{* * *}$ \\
Año t+3 & $-7,83 \mathrm{e}-06^{* * *}$ & $-0,0038^{* * *}$ & $0,00001^{* * *}$ & & $0,00003^{* * *}$ \\
Año t+4 & $-0,00001^{* * *}$ & $-0,0058^{* * *}$ & & & \\
\hline LR Chi 2 (7) & $14438,15^{* * *}$ & $42430,27^{* * *}$ & & & $164635,94^{* * *}$ \\
LR Chi 2 (6) & & & $117554,36^{* * *}$ & $30831,84^{* * *}$ & \\
Muestra & 286 & 996 & 635 & 432 & 635 \\
\hline
\end{tabular}

*** significativo al $1 \%$

** significativo al $5 \%$ 
Cuadro 5b. Regresiones Logit Binomial en el panel

\begin{tabular}{l|c|c|c|c|c}
\hline Logit binomial en el panel & \multicolumn{5}{|c}{ Pasa al desempleo o a la inactividad } \\
\hline Base = sigue como THR & $1998-2002$ & $2002-2006$ & $2007-2010$ & $2004-2006$ & $2007-2010$ \\
\hline Nivel de educación & $0,0726^{* * *}$ & $0,0496^{* * *}$ & $0,1931^{* * *}$ & $0,0732^{* * *}$ & $0,1912^{* * *}$ \\
Edad promedio & $-0,2603^{* * *}$ & $-0,1213^{* * *}$ & $-0,2787^{* * *}$ & $-0,0928^{* * *}$ & $-0,2699^{* * *}$ \\
Jefe de hogar & $0,9846^{* * *}$ & $-0,3497^{* * *}$ & $-1,1763^{* * *}$ & $-21,07220$ & $1,1747^{* * *}$ \\
Lengua materna & $\mathrm{n}, \mathrm{d}$, & $\mathrm{n}, \mathrm{d}$, & $\mathrm{n}, \mathrm{d}$, & $0,2236^{* * *}$ & $-0,1001^{* * *}$ \\
Año t+1 & $1,6835^{* * *}$ & $-0,0220^{* * *}$ & $-0,0851^{* * *}$ & $-0,0454^{* * *}$ & $-0,0953^{* * *}$ \\
Año t+2 & $1,0862^{* * *}$ & $-0,2188^{* * *}$ & $0,1854^{* * *}$ & $-0,2534^{* * *}$ & $0,1674^{* * *}$ \\
Año t+3 & $1,2604^{* * *}$ & $-0,1977^{* * *}$ & $0,3018^{* * *}$ & & $0,2745^{* * *}$ \\
Año t+4 & $1,5646^{* * *}$ & $-0,3303^{* * *}$ & & & \\
\hline Efectos marginales & $1998-2002$ & $2002-2006$ & $2007-2010$ & $2004-2006$ & $2007-2010$ \\
\hline Nivel de educación & $0,00008^{* * *}$ & $0,0010^{* * *}$ & $0,00003^{* * *}$ & 0,0002 & $0,00004^{* * *}$ \\
Edad promedio & $-0,0003^{* * *}$ & $-0,0026^{* * *}$ & $-0,00004^{* * *}$ & $-0,0002$ & $-0,00005^{* * *}$ \\
Jefe de hogar & $0,0017^{* * *}$ & $-0,0065^{* * *}$ & $-0,0001^{* * *}$ & $-0,0568^{* * *}$ & $-0,0001^{* * *}$ \\
Lengua materna & $\mathrm{n}, \mathrm{d}$, & $\mathrm{n}, \mathrm{d}$, & $\mathrm{n}, \mathrm{d}$, & 0,0006 & $-0,00002^{* * *}$ \\
Año t+1 & $0,0042^{* * *}$ & $-0,0005^{* * *}$ & $-0,00001^{* * *}$ & $-0,0001$ & $-0,00002^{* * *}$ \\
Ańo t+2 & $0,0019^{* * *}$ & $-0,0044^{* * *}$ & $0,00003^{* * *}$ & $-0,0006$ & $0,00003^{* * *}$ \\
Año t+3 & $0,0018^{* * *}$ & $-0,0039^{* * *}$ & $0,00005^{* * *}$ & & $0,00006^{* * *}$ \\
Año t+4 & $0,0024^{* * *}$ & $-0,0064^{* * *}$ & & & \\
\hline LR Chi 2 (7) & $22993,48^{* * *}$ & $39525,24^{* * *}$ & & & \\
LR Chi 2 (6) & & & $54387,10^{* * *}$ & $24535,18^{* * *}$ & $54506,70^{* * *}$ \\
Muestra & 325 & 1067 & 522 & 461 & 522 \\
\hline
\end{tabular}

*** significativo al $1 \%$

** significativo al $5 \%$

\section{CONCLUSIONES}

El objetivo de este artículo era analizar las transiciones desde el trabajo del hogar remunerado hacia otras ocupaciones, el desempleo y la inactividad, así como encontrar los determinantes de estas transiciones. Para ello empleamos los datos panel de la ENAHO para los periodos 1998-2002, 2002-2006 y 2007-2011, lo cual además nos permitió analizar los efectos del ciclo económico sobre la movilidad en el mercado del trabajo del hogar remunerado.

Encontramos que un mayor nivel de educación está siempre asociado a una salida del THR hacia otra ocupación, si bien en la mayoría de los casos es hacia el trabajo independiente. A la vez, un mayor nivel de educación está asociado a una mayor probabilidad de salir hacia la inactividad — la salida hacia el desempleo es casi inexistente- aspecto en el cual las trabajadoras del hogar se diferencian de las mujeres ocupadas en general. Para ellas un aumento en su nivel de educación aumenta la probabilidad de buscar otro trabajo, pero reduce la probabilidad de salir de la fuerza laboral. Una explicación posible de esta diferencia podría estar en una menor disposición de las trabajadoras a permanecer en esta ocupación, con relación a permanecer en su hogar. Esto solamente sería 
posible en el caso de tener otra fuente de ingresos, lo cual amerita una mayor investigación sobre este punto en un trabajo posterior.

En el caso de la edad, encontramos que la movilidad desde el THR hacia otra ocupación se reduce con la edad para los periodos 2002-2006 y 2007-2010, lo cual es consistente con una menor probabilidad de salir de la fuerza laboral para todos los paneles. Es decir, las trabajadoras del hogar que permanecen en dicha ocupación son las de mayor edad, las cuales — al contrario que las mujeres en general — se mantienen en la fuerza laboral hasta edades avanzadas.

Finalmente, encontramos que durante los periodos 1998-2002 y 2007-2010, en los cuales hay caídas importantes del producto, tanto la salida hacia otra ocupación como hacia la inactividad aumenta. Así, si bien la educación permite salir del THR hacia otras ocupaciones, esto no implica encontrar un puesto de trabajo mejor en los periodos en los cuales hay crisis económica. Es decir, el ciclo influye no solamente en la probabilidad de encontrar un empleo alternativo, sino en la probabilidad de perder el actual. En el caso del panel 2007-2010, la probabilidad de salir del THR aumenta aún después de la crisis, y la salida hacia otras ocupaciones es mayor en el año luego de la caída del PIB de 2009. Esto estaría relacionado a caídas en la demanda por los servicios de las trabajadoras del hogar, que en su mayoría saldrán hacia el trabajo independiente. Asimismo, mientras la salida hacia la inactividad aumenta durante todo el periodo para las mujeres en general, en el caso de las trabajadoras del hogar solamente aumenta a partir de la crisis global del año 2009.

Anexo 1. Características de las encuestas empleadas en esta investigación

\begin{tabular}{l|c|c|c|c}
\hline & Marco muestral & Periodo & Tamańo de muestra & Hogares Panel \\
\hline Panel 1998-2002 & Censo de 1993 & mayo-junio 1998 & 3681 hogares & 2957 hogares \\
& & noviembre-diciembre 1999 & 3296 hogares & 3015 hogares \\
& & octubre-diciembre 2000 & 3559 hogares & 1238 hogares \\
& & noviembre-diciembre 2001 & 16277 hogares & 2896 hogares \\
& & octubre-diciembre 2002 & 4659 hogares & 4659 hogares \\
\hline Panel 2002-2006 & Censo de 1993 & 2002 - IV trimestre & 6257 hogares & \\
& & $2003-$ mayo-diciembre & 4217 hogares & \\
& & 2004 anual & 6490 hogares & \\
& & 2005 anual & 6778 hogares & 4026 hogares \\
\hline Panel 2007-2011 & Censo 2005 & 2006 anual & 6593 hogares & \\
& & 2007 anual & 7767 hogares & \\
& & 2008 anual & 7730 hogares & \\
& & 2009 anual & 7595 hogares & 1129 hogares \\
\hline
\end{tabular}

Fuente: Elaboración de la autora a partir de las fichas técnicas de las ENAHO Panel 1998-2002, 2002-2006 y $2007-2011$. 
Anexo 2. Nivel de educación y edad promedio según transición laboral

\begin{tabular}{l|c|c|c|c}
\hline \multicolumn{1}{c|}{ 1998-2001 } & Siguen en THR & Otra ocupación & Desempleo & Inactividad \\
\hline Edad promedio & 26,1 & 20,0 & n.d. & 44,4 \\
Nivel de educación & 3,9 & 6,0 & n.d. & 4,2 \\
\% área urbana & 92,20 & 100,00 & n.d. & 100,00 \\
\hline
\end{tabular}

Fuente: Elaboración de la autora a partir de la ENAHO Panel 1998-2002. Datos de inicio del periodo

\begin{tabular}{l|c|c|c|c}
\hline \multicolumn{1}{c|}{ 2002-2006 } & Siguen en THR & Otra ocupación & Desempleo & Inactividad \\
\hline Edad promedio & 37,9 & 35,1 & 34,0 & 31,7 \\
Nivel de educación & 4,6 & 4,9 & 4,3 & 4,2 \\
\% área urbana & 97,7 & 97,6 & 100,0 & 83,3 \\
\hline
\end{tabular}

Fuente: Elaboración de la autora a partir de la ENAHO Panel 2002-2006. Datos de inicio del periodo

\begin{tabular}{l|c|c|c|c}
\hline \multicolumn{1}{c|}{ 2004-2006 } & Siguen en THR & Otra ocupación & Desempleo & Inactividad \\
\hline \% lengua nativa & 34,7 & 29,9 & 0,0 & 13,6 \\
Edad promedio & 36,0 & 35,6 & 29,9 & 37,8 \\
Nivel de educación & 4,8 & 5,0 & 4,0 & 4,6 \\
\% área urbana & 97,4 & 95,3 & 100,0 & 92,0 \\
\hline
\end{tabular}

Fuente: Elaboración de la autora a partir de la ENAHO Panel 2004-2006. Datos de inicio del periodo.

\begin{tabular}{l|c|c|c|c}
\hline \multicolumn{1}{c|}{ 2007-2011 } & Siguen en THR & Otra ocupación & Desempleo & Inactividad \\
\hline \% lengua nativa & 25,6 & 20,4 & 0,0 & 0,0 \\
Edad promedio & 37,4 & 32,4 & 19,0 & 27,2 \\
Nivel de educación & 5,1 & 5,3 & 5,3 & 6,0 \\
\% área urbana & 100,0 & 96,4 & 100,0 & 100,0 \\
\hline
\end{tabular}

Fuente: Elaboración de la autora a partir de la ENAHO Panel 2004-2006. Datos de inicio del periodo.

Niveles de educación: 1: sin nivel; 2: inicial; 3: primaria incompleta; 4: primaria completa; 5: secundaria incompleta; 6: secundaria completa; 7: superior no universitaria incompleta; 8: superior no universitaria completa; 9: superior universitaria incompleta; 10: superior universitaria completa; 11: posgrado 


\section{Anexo 3. Transiciones por paneles urbano y rural}

urbano

1998-2002

\begin{tabular}{l|c|c|c|c|c|r|r}
\hline \multicolumn{2}{c|}{} & \multicolumn{7}{c}{ 2002 } \\
\cline { 3 - 8 } \multicolumn{2}{c|}{} & Trab. del hogar & Otras ocup. & Desemp. & Inactivos & Total & Población \\
\hline \multirow{4}{*}{1998} & Trabajador del hogar & 25,7 & 24,9 & 0,0 & 49,4 & 100,0 & 22273 \\
& Otras ocupaciones & 0,5 & 76,7 & 2,7 & 20,1 & 100,0 & 386589 \\
& Desempleados & 4,2 & 51,8 & 0,0 & 44,0 & 100,0 & 31229 \\
& Inactivos & 2,0 & 36,4 & 3,1 & 58,5 & 100,0 & 250006 \\
\cline { 2 - 8 } & Población & 14106 & 409051 & 18340 & 248600 & 100,0 & 690097 \\
\hline
\end{tabular}

Fuente: ENAHO-INEI Panel 1998-2002

Rural

\begin{tabular}{l|c|c|c|c|c|r|r}
\hline \multicolumn{2}{c|}{} & \multicolumn{7}{c}{$\mathbf{2 0 0 2}$} \\
\cline { 3 - 8 } \multicolumn{2}{c|}{} & Trab. del hogar & Otras ocup. & Desemp. & Inactivos & Total & Población \\
\hline \multirow{4}{*}{1998} & Trabajador del hogar & 0,0 & 0,0 & 100,0 & 0,0 & 100,0 & 229 \\
& Otras ocupaciones & 0,0 & 89,5 & 0,7 & 9,8 & 100,0 & 172478 \\
& Desempleados & 0,0 & 45,3 & 33,5 & 21,2 & 100,0 & 1730 \\
& Inactivos & 0,6 & 46,4 & 4,6 & 48,4 & 100,0 & 57135 \\
\cline { 2 - 8 } & Población & 366 & 181748 & 4551 & 44907 & 100,0 & 231572 \\
\hline
\end{tabular}

Fuente: ENAHO-INEI Panel 1998-2002.

2002-2006

Urbano

2006

\begin{tabular}{ll|c|c|c|c|c|r|}
\cline { 2 - 7 } & Trab. del hogar & Otras ocup. & Desemp. & Inactivos & Total & Población \\
\hline Trabajador del hogar & 40,0 & 33,6 & 3,6 & 22,8 & 100,0 & 220811 \\
Otras ocupaciones & 1,1 & 84,5 & 1,9 & 12,5 & 100,0 & 6180463 \\
Desempleados & 1,5 & 55,4 & 8,4 & 34,7 & 100,0 & 421450 \\
Inactivos & 3,1 & 37,9 & 5,5 & 53,5 & 100,0 & 3913403 \\
\hline Población & 279624 & 7012934 & 379352 & 3064217 & 100,0 & 10736027 \\
\hline
\end{tabular}

Fuente: ENAHO-INEI Panel 2002.2006.

Rural

\begin{tabular}{l|l|c|c|c|c|r|r}
\hline \multicolumn{2}{c|}{} & \multicolumn{7}{c}{2006} \\
\cline { 3 - 8 } \multicolumn{2}{c|}{} & Trab. del hogar & Otras ocup. & Desemp. & Inactivos & Total & Población \\
\hline \multirow{3}{*}{2002} & Trabajador del hogar & 8,0 & 13,0 & 0,0 & 79,0 & 100,0 & 6479 \\
& Otras ocupaciones & 0,1 & 91,2 & 0,2 & 8,4 & 100,0 & 3258313 \\
& Desempleados & 11,0 & 70,6 & 11,7 & 6,7 & 100,0 & 23522 \\
& Inactivos & 0,9 & 59,0 & 0,7 & 39,4 & 100,0 & 688573 \\
\cline { 2 - 7 } & Población & 13344 & 3395237 & 14726 & 553580 & 100,0 & 3976887 \\
\hline
\end{tabular}

Fuente: ENAHO-INEI Panel 2002-2006. 
2007-2011

Urbano

\begin{tabular}{l|c|c|c|c|c|c|r}
\hline \multicolumn{2}{c|}{} & \multicolumn{6}{c}{2010} \\
\cline { 3 - 8 } \multicolumn{2}{c|}{} & Trab. del hogar & Otras ocup. & Desemp. & Inactivos & Total & Población \\
\hline \multirow{3}{*}{2007} & Trabajador del hogar & 22,8 & 56,6 & 2,0 & 18,6 & 100,0 & 395673 \\
& Otras ocupaciones & 1,1 & 85,8 & 1,7 & 11,4 & 100,0 & 13060747 \\
& Desempleados & 0,0 & 66,7 & 4,2 & 29,1 & 100,0 & 768118 \\
& Inactivos & 3,1 & 36,0 & 6,3 & 54,6 & 100,0 & 6253476 \\
\cline { 2 - 8 } & Población & 423118 & 14203039 & 659232 & 5192625 & 100,0 & 20478014 \\
\hline
\end{tabular}

Fuente: ENAHO-INEI Panel 2007-2010.

Rural

\begin{tabular}{l|l|c|c|c|c|r|r}
\hline \multicolumn{2}{c|}{} & \multicolumn{7}{c}{2010} \\
\cline { 3 - 8 } \multicolumn{2}{c|}{2007} & Trab. del hogar & Otras ocup. & Desemp. & Inactivos & Total & Población \\
\hline \multirow{4}{*}{20,0} & 100,0 & 0,0 & 0,0 & 100,0 & 8337 \\
& Trabajador del hogar & 0,0 & 89,2 & 0,1 & 10,5 & 100,0 & 4260358 \\
& Otras ocupaciones & 0,2 & 63,5 & 0,0 & 36,5 & 100,0 & 13124 \\
& Desempleados & 0,0 & 54,3 & 2,2 & 40,9 & 100,0 & 572048 \\
\cline { 2 - 8 } & Inactivos & 2,6 & 4125792 & 16968 & 687504 & 100,0 & 4853867 \\
\cline { 2 - 8 } & Población & 23603 & & &
\end{tabular}

Fuente: ENAHO-INEI Panel 2007-2010.

\section{Anexo 4. Variación del PBI real, 1998-2011, base 1994=100}

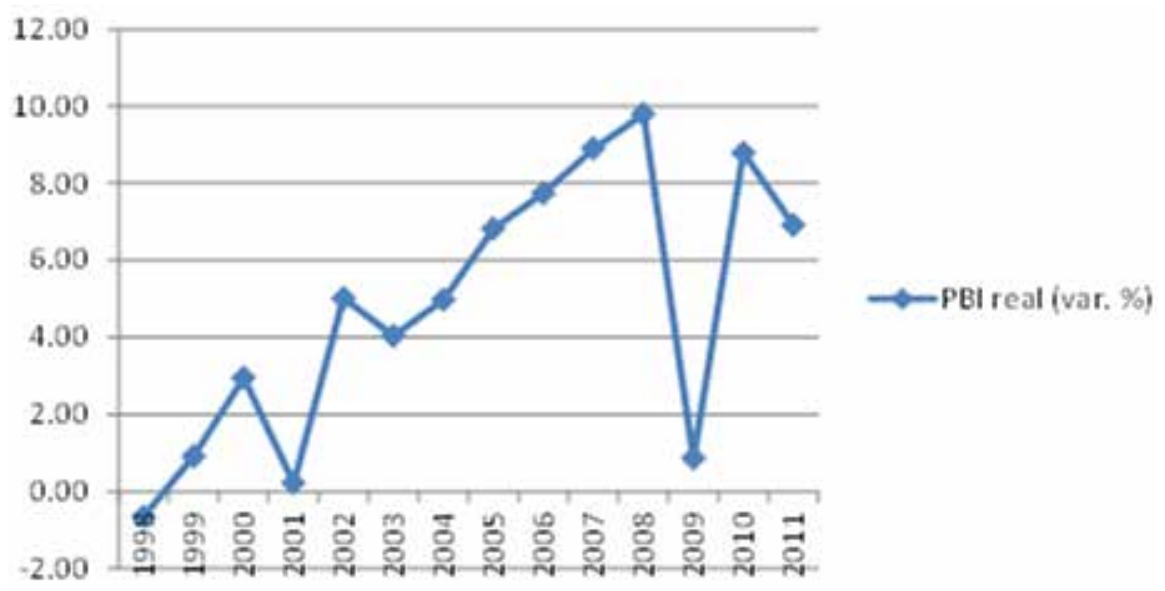




\section{Anexo 5. Transiciones entre ocupaciones, paneles parciales y total 1998-2002}

\begin{tabular}{c|l|c|c|c|c|c|c|r}
\hline \multicolumn{2}{c|}{} & \multicolumn{7}{c}{1999} \\
\cline { 3 - 9 } \multicolumn{2}{c|}{} & Empleador & Independiente & Empleado & Obrero & TFNR & Trab. hogar & Población \\
\hline \multirow{3}{*}{1998} & Empleador & 30,1 & 19,9 & 22,8 & 18,5 & 8,7 & 0,0 & 43000 \\
& Trab. independiente & 5,0 & 80,2 & 4,2 & 8,2 & 2,4 & 0,0 & 201475 \\
& Empleado & 0,0 & 11,4 & 70,0 & 17,1 & 1,5 & 0,0 & 75183 \\
& Obrero & 0,7 & 18,4 & 7,0 & 71,2 & 2,7 & 0,0 & 120512 \\
& TFNR & 2,7 & 18,2 & 3,1 & 12,5 & 61,0 & 2,5 & 103759 \\
& Trabajador del hogar & 0,0 & 42,3 & 0,0 & 0,0 & 0,0 & 57,7 & 9644 \\
\cline { 2 - 9 } & Población & 26695 & 223781 & 82629 & 136194 & 76142 & 8132 & 553573 \\
\hline
\end{tabular}

Fuente: ENAHO-INEI Panel 1998-1999

\begin{tabular}{c|l|c|c|c|c|c|c|r}
\hline \multicolumn{2}{c|}{} & \multicolumn{7}{c}{2000} \\
\cline { 3 - 9 } \multicolumn{2}{c|}{} & Empleador & Independiente & Empleado & Obrero & TFNR & Trab. hogar & Población \\
\hline \multirow{3}{*}{1998} & Empleador & 38,3 & 20,5 & 15,2 & 18,9 & 7,1 & 0,0 & 40436 \\
& Trab. independiente & 3,8 & 79,2 & 2,9 & 6,5 & 5,9 & 1,7 & 205772 \\
& Empleado & 5,5 & 16,2 & 62,9 & 14,0 & 1,4 & 0,0 & 78287 \\
& Obrero & 1,8 & $15,0^{*}$ & 12,0 & 64,0 & 7,2 & 0,0 & 127971 \\
& TFNR & 4,2 & 24,0 & 0,0 & 8,2 & 60,7 & 2,9 & 87254 \\
& Trabajador del hogar & 0,0 & 0,0 & 2,2 & 20,1 & 9,8 & 67,9 & 12753 \\
\cline { 2 - 9 } & Población & 33754 & $224040^{*}$ & 76941 & 123578 & 79520 & 14640 & 552473 \\
\hline
\end{tabular}

Fuente: ENAHO-INEI Panel 1998-2000.

* El rubro «otros» de trabajos no especificados ha sido incluido en la categoría «independiente».

\begin{tabular}{c|c|c|c|c|c|c|r|r}
\hline \multicolumn{2}{c|}{} & \multicolumn{7}{c}{ 2001 } \\
\cline { 3 - 9 } \multicolumn{2}{c|}{} & Empleador & Independiente & Empleado & Obrero & TFNR & Trab. hogar & Población \\
\hline \multirow{3}{*}{1998} & 24,8 & 30,4 & 24,8 & 13,4 & 6,6 & 0,0 & 43194 \\
& Empleador & 8,4 & 76,4 & 5,6 & 4,0 & 1,9 & 3,7 & 195805 \\
& Trab. independiente & 2,3 & 37,0 & 47,8 & 11,2 & 1,7 & 0,0 & 74935 \\
& Empleado & 2,4 & 10,6 & 6,0 & 74,4 & 10,6 & 0,0 & 126510 \\
& Obrero & 4,4 & 11,3 & 9,8 & 9,0 & 62,4 & 3,1 & 83291 \\
& TFNR & 0,0 & 38,0 & 0,0 & 0,0 & 18,5 & 43,5 & 6744 \\
\hline & Trabajador del hogar & 35626 & 215782 & 73275 & 123622 & 69498 & 12676 & 530479 \\
\cline { 2 - 9 } & Población & & & & & &
\end{tabular}

Fuente: ENAHO-INEI Panel 1998-2001.

\begin{tabular}{l|l|c|c|c|c|c|c|r}
\hline \multicolumn{2}{c|}{} & \multicolumn{7}{c}{2002} \\
\cline { 3 - 9 } \multicolumn{2}{c|}{} & Empleador & Independiente & Empleado & Obrero & TFNR & Trab. hogar & Población \\
\hline \multirow{3}{*}{1998} & Empleador & 28,6 & 27,6 & 21,7 & 18,5 & 3,6 & 0,0 & 40160 \\
& Trab. independiente & 8,4 & 74,3 & 5,8 & 7,9 & 2,5 & 1,1 & 182912 \\
& Empleado & 0,0 & 25,3 & 65,4 & 8,0 & 1,3 & 0,0 & 67252 \\
& Obrero & 1,9 & $15,4^{*}$ & 9,8 & 63,8 & 9,1 & 0,0 & 114 \\
& TFNR & 1,1 & 15,8 & 5,0 & 17,7 & 56,6 & 3,8 & 79127 \\
& Trabajador del hogar & 0,0 & 21,6 & 22,7 & 0,0 & 10,0 & 45,7 & 12524 \\
\hline & Población & 29850 & $196827^{*}$ & 81275 & 113934 & 63331 & 10770 & 495987 \\
\hline
\end{tabular}

Fuente: ENAHO-INEI Panel 1998-2002

* El rubro «otros» de trabajos no especificados ha sido incluido en la categoría «independiente». 


\section{Anexo 6. Transiciones entre ocupaciones, paneles parciales y total 2002-2006}

\begin{tabular}{l|l|c|c|c|c|c|c|c}
\hline \multicolumn{2}{c|}{} & \multicolumn{9}{c}{2003} \\
\cline { 3 - 9 } \multicolumn{2}{c|}{} & Empleador & Independiente & Empleado & Obrero & TFNR & Trab. Hogar & Población \\
\hline \multirow{3}{*}{2002} & Empleador & 43,3 & 47,5 & 0,8 & 4,4 & 4,0 & 0,0 & 463545 \\
& Trab. independiente & 5,6 & $75,5^{*}$ & 2,3 & 6,7 & 9,5 & 0,4 & $2988037^{*}$ \\
& Empleado & 1,1 & $8,6^{*}$ & 78,9 & 8,4 & 2,3 & 0,7 & 1310615 \\
& Obrero & 2,6 & $16,9^{*}$ & 7,5 & 63,5 & 8,4 & 1,1 & 1274039 \\
& TFNR & 1,3 & $15,0^{*}$ & 2,3 & 6,8 & 73,1 & 1,5 & 1589038 \\
& Trabajador del hogar & 0,6 & 10,6 & 11,9 & 10,2 & 6,7 & 60,0 & 157153 \\
\cline { 2 - 9 } & Población & 437877 & $3060924^{*}$ & 1256780 & 1265190 & 1609339 & 152317 & 7782427 \\
\hline
\end{tabular}

Fuente: ENAHO-INEI Panel 2002-2003

* El rubro «otros» de trabajos no especificados ha sido incluido en la categoría «independiente».

\begin{tabular}{|c|c|c|c|c|c|c|c|c|}
\hline & \multicolumn{7}{|c|}{2004} \\
\hline & & Empleador & Independiente & Empleado & Obrero & TFNR & Trab. Hogar & Población \\
\hline \multirow{7}{*}{2002} & Empleador & 45,4 & $40,4^{*}$ & 2,8 & 7,5 & 3,9 & 0,0 & 633073 \\
\hline & Trab. independiente & 5,5 & $72,3^{*}$ & 5,3 & 11,2 & 7,8 & 0,7 & $4094239^{*}$ \\
\hline & Empleado & 2,7 & $11,9^{*}$ & 74,5 & 7,9 & 2,6 & 0,4 & 1680476 \\
\hline & Obrero & 2,8 & $22,5^{*}$ & 10,7 & 54,7 & 8,3 & 1,0 & 1755424 \\
\hline & TFNR & 1,4 & $15,7^{*}$ & 2,9 & 10,0 & 68,3 & 1,7 & 2233701 \\
\hline & Trabajador del hogar & 0,0 & 9,5 & 21,6 & 8,8 & 11,1 & 49,0 & 215582 \\
\hline & Población & 702847 & $4185206^{*}$ & 1728700 & 1716179 & 2088856 & 189708 & 10611496 \\
\hline
\end{tabular}

Fuente: ENAHO-INEI Panel 2002-2004

* El rubro «otros» de trabajos no especificados ha sido incluido en la categoría «independiente».

\begin{tabular}{c|l|c|c|c|c|c|r|r}
\hline \multicolumn{2}{c|}{} & \multicolumn{9}{c}{2005} \\
\cline { 3 - 9 } \multicolumn{2}{c|}{} & Empleador & Independiente & Empleado & Obrero & TFNR & Trab. Hogar & Población \\
\hline \multirow{3}{*}{2002} & Empleador & 35,6 & 50,0 & 7,5 & 5,0 & 1,9 & 0,0 & 537323 \\
& Trab. independiente & 7,0 & $73,3^{*}$ & 4,2 & 7,5 & 7,0 & 1,0 & $3633739^{*}$ \\
& Empleado & 2,3 & $10,4^{*}$ & 72,0 & 11,2 & 2,5 & 1,6 & 1384276 \\
& Obrero & 4,6 & $24,6^{*}$ & 11,4 & 51,3 & 7,5 & 0,6 & 1463445 \\
& TFNR & 2,1 & $16,9^{*}$ & 3,4 & 10,6 & 65,3 & 1,7 & 1923424 \\
& Trabajador del hogar & 0,6 & $16,1^{*}$ & 9,2 & 8,9 & 6,4 & 58,8 & 167737 \\
\cline { 2 - 9 } & Población & 586641 & $3785658^{*}$ & 1438074 & 1425832 & 1676784 & 196956 & 9109945 \\
\hline
\end{tabular}

Fuente: ENAHO-INEI Panel 2002-2005

* El rubro «otros» de trabajos no especificados ha sido incluido en la categoría «independiente».

\begin{tabular}{c|l|c|c|c|c|c|r|r}
\hline \multicolumn{2}{c|}{} & \multicolumn{9}{c}{2006} \\
\cline { 2 - 9 } \multicolumn{2}{c|}{} & Empleador & Independiente & Empleado & Obrero & TFNR & Trab. Hogar & Población \\
\hline \multirow{3}{*}{2002} & Empleador & 42,8 & $41,9^{*}$ & 4,4 & 8,2 & 2,6 & 0,1 & 540884 \\
& Trab. independiente & 8,4 & $70,5^{*}$ & 3,9 & 8,7 & 8,7 & 7,8 & $3538645^{*}$ \\
& Empleado & 1,3 & $12,9^{*}$ & 72,5 & 9,0 & 3,1 & 1,1 & 1435677 \\
& Obrero & 4,3 & $23,3^{*}$ & 11,0 & 53,3 & 6,7 & 1,4 & 1427329 \\
& TFNR & 0,8 & $20,3^{*}$ & 4,0 & 13,6 & 60,2 & 1,1 & 1811147 \\
& Trabajador del hogar & 5,5 & 10,1 & 7,2 & 13,0 & 9,9 & 54,3 & 169887 \\
\cline { 2 - 9 } & Población & 586641 & $3785658^{*}$ & 1438074 & 1425832 & 1676784 & 196957 & 8923569 \\
\hline
\end{tabular}

Fuente: ENAHO-INEI Panel 2002-2006

* El rubro «otros» de trabajos no especificados ha sido incluido en la categoría «independiente». 
Anexo 7. Transiciones entre ocupaciones, paneles parciales y total 2007-2011

\begin{tabular}{l|l|c|c|c|c|c|c|c}
\hline \multicolumn{2}{c|}{} & \multicolumn{9}{c}{ 2008 } \\
\cline { 3 - 9 } \multicolumn{2}{c|}{} & Empleador & Independiente & Empleado & Obrero & TFNR & Trab. Hogar & Población \\
\hline \multirow{3}{*}{2007} & 45,2 & 37,5 & 4,8 & 7,1 & 5,1 & 0,3 & 976829 \\
& Empleador & 6,2 & $73,5^{*}$ & 6,2 & 7,0 & 6,0 & 1,1 & $5933000^{*}$ \\
& Trab. independiente & 1,9 & $8,2^{*}$ & 77,7 & 8,6 & 2,3 & 1,3 & 3542785 \\
& Empleado & 2,5 & $17,3^{*}$ & 7,9 & 64,2 & 6,9 & 1,2 & 2798433 \\
& Obrero & 1,5 & $16,2^{*}$ & 5,1 & 8,3 & 67,6 & 1,3 & 2691921 \\
& TFNR & 0,0 & $10,2^{*}$ & 18,0 & 7,2 & 4,2 & 60,4 & 462185 \\
\hline & Trabajador del hogar & 989437 & $5983376^{*}$ & 3610915 & 2842133 & 2515677 & 462615 & 16405153 \\
\cline { 2 - 9 } & Población & & &
\end{tabular}

Fuente: ENAHO-INEI Panel 2007-2008

* El rubro «otros» de trabajos no especificados ha sido incluido en la categoría «independiente».

\begin{tabular}{l|l|c|c|c|c|c|c|c}
\hline \multicolumn{2}{c|}{} & \multicolumn{9}{c}{ 2009 } \\
\cline { 3 - 9 } \multicolumn{2}{c|}{} & Empleador & Independiente & Empleado & Obrero & TFNR & Trab. Hogar & Población \\
\hline \multirow{3}{*}{2007} & 44,6 & $38,0^{*}$ & 5,3 & 8,0 & 3,7 & 0,4 & 888684 \\
& Empleador & 6,5 & $73,5^{*}$ & 5,1 & 7,4 & 6,5 & 1,0 & $5482368^{*}$ \\
& Trab. independiente & 1,7 & $7,3^{*}$ & 79,8 & 8,5 & 2,0 & 0,7 & 2845218 \\
& Empleado & 3,0 & $19,7^{*}$ & 8,5 & 59,1 & 8,1 & 1,6 & 2437391 \\
& Obrero & 1,3 & $17,2^{*}$ & 5,5 & 11,7 & 63,7 & 0,6 & 2535926 \\
& TFNR & 0,1 & 12,0 & 15,5 & 13,1 & 6,8 & 52,5 & 423202 \\
\hline & Trabajador del hogar & 910029 & $5542131^{*}$ & 3011157 & 2513273 & 2289061 & 356338 & 14612789 \\
\cline { 2 - 9 } & Población & & & & &
\end{tabular}

Fuente: ENAHO-INEI Panel 2007-2009

* El rubro «otros» de trabajos no especificados ha sido incluido en la categoría «independiente».

\begin{tabular}{c|l|c|c|c|c|c|c|c}
\hline \multicolumn{2}{c|}{} & \multicolumn{9}{c}{2010} \\
\cline { 3 - 9 } \multicolumn{2}{c|}{} & Empleador & Independiente & Empleado & Obrero & TFNR & Trab. Hogar & Población \\
\hline \multirow{3}{*}{2007} & Empleador & 41,7 & $38,6^{*}$ & 8,7 & 6,3 & 4,7 & 0,0 & 879230 \\
& Trab. independiente & 6,1 & $70,7^{*}$ & 6,2 & 8,6 & 7,5 & 0,9 & $5828423^{*}$ \\
& Empleado & 1,9 & $6,6^{*}$ & 72,3 & 9,5 & 1,7 & 1,0 & 3045258 \\
& Obrero & 3,2 & $19,8^{*}$ & 9,2 & 57,8 & 5,8 & 0,2 & 2785900 \\
& TFNR & 2,5 & $18,8^{*}$ & 6,9 & 11,7 & 58,1 & 2,0 & 2557692 \\
& Trabajador del hogar & 0,7 & 20,7 & 17,6 & 10,7 & 5,7 & 44,6 & 383752 \\
\cline { 2 - 9 } & Población & 933695 & $5774036^{*}$ & 3464016 & 2794133 & 2200584 & 52561 & 15480255 \\
\hline
\end{tabular}

Fuente: ENAHO-INEI Panel 2007-2010

* El rubro "otros» de trabajos no especificados ha sido incluido en la categoría «independiente».

\begin{tabular}{l|l|c|c|c|c|c|c|r}
\hline \multicolumn{2}{c|}{} & \multicolumn{9}{c}{2011} \\
\cline { 2 - 8 } \multicolumn{2}{c|}{} & Empleador & Independiente & Empleado & Obrero & TFNR & Trab. Hogar & Población \\
\hline \multirow{5}{*}{2007} & 35,7 & 38,5 & 7,7 & 10,9 & 6,7 & 0,5 & 1088454 \\
& Empleador & 6,0 & $71,3^{*}$ & 6,6 & 8,5 & 6,5 & 1,1 & 5953831 \\
& Trab. independiente & 3,2 & 9,2 & 75,2 & 9,4 & 2,1 & 0,9 & 3366529 \\
& Empleado & 3,7 & $28,1^{*}$ & 12,4 & 51,9 & 3,6 & 0,3 & 2860693 \\
& Obrero & 3,9 & $20,4^{*}$ & 8,5 & 9,3 & 56,0 & 1,9 & 2711826 \\
& TFNR & 0,0 & $26,1^{*}$ & 21,3 & 22,7 & 0,0 & 29,9 & 337434 \\
\hline
\end{tabular}

Fuente: ENAHO-INEI Panel 2007-2011

* El rubro «otros» de trabajos no especificados ha sido incluido en la categoría «independiente». 


\section{Anexo 8. Determinantes de las transiciones laborales de las mujeres en general}

\begin{tabular}{|c|c|c|c|c|}
\hline \multirow{2}{*}{$\begin{array}{l}\text { Logit binomial en el panel } \\
\text { Base }=\text { sigue como THR }\end{array}$} & \multicolumn{4}{|c|}{ Pasa al desempleo } \\
\hline & $1998-2002$ & $2002-2006$ & $2007-2010$ & $2007-2010$ \\
\hline Nivel de educación & $0,2102^{* * *}$ & $-0,3136^{* * *}$ & $-0,5192^{* * *}$ & $-0,5207^{* * *}$ \\
\hline Edad promedio & $0,1133^{* * *}$ & $-0,0595^{* * *}$ & $0,8535^{* * *}$ & $0,8556^{* * *}$ \\
\hline Jefe de hogar & $-1,0863^{* * *}$ & $-0,3669^{* * *}$ & $0,3107^{* * *}$ & $0,3011^{* * *}$ \\
\hline Lengua materna nativa & $\mathrm{n}, \mathrm{d}$, & $\mathrm{n}, \mathrm{d}$, & $\mathrm{n}, \mathrm{d}$ & $-0,0532^{* * *}$ \\
\hline Año $t+1$ & $-0,7357^{* * *}$ & $0,0323^{* * *}$ & $-0,8587^{* * *}$ & $-0,8624^{* * *}$ \\
\hline Año $t+2$ & $-0,6048^{* * *}$ & $-0,0672^{* * *}$ & $-1,6393^{* * *}$ & $-1,6454^{* * *}$ \\
\hline Año $t+3$ & $-0,7926^{* * *}$ & $-0,2516^{* * *}$ & $-2,7682^{* * *}$ & $-2,7745^{* * *}$ \\
\hline Año $t+4$ & $-0,9015^{* * *}$ & $-0,1785^{* * *}$ & & \\
\hline Efectos marginales & $1998-2002$ & $2002-2006$ & $2007-2010$ & $2007-2010$ \\
\hline Nivel de educación & $0,0044^{* * *}$ & $-0,0054^{* * *}$ & $-1,02 \mathrm{e}-09^{* * *}$ & $-1,21 \mathrm{e}-09^{* * *}$ \\
\hline Edad promedio & $0,0024^{* * *}$ & $-0,0010^{* * *}$ & $1,67 e-09^{* * *}$ & $1,98 \mathrm{e}-09^{* * *}$ \\
\hline Jefe de hogar & $-0,0295^{* * *}$ & $-0,0054^{* * *}$ & $2,09 \mathrm{e}-10^{* * *}$ & $-7,54 \mathrm{e}-06^{* * *}$ \\
\hline Lengua materna nativa & $\mathrm{n}, \mathrm{d}$ & $\mathrm{n}, \mathrm{d}$ & $\mathrm{n}, \mathrm{d}$ & $2,38 \mathrm{e}-10^{* * *}$ \\
\hline Año $t+1$ & $-0,0212^{* * *}$ & $0,0006^{* * *}$ & $-8,21 \mathrm{e}-10^{* * *}$ & $-9,66 \mathrm{e}-10^{* * *}$ \\
\hline Año $t+2$ & $-0,0157^{* * *}$ & $-0,0011^{* * *}$ & $-2,09 \mathrm{e}-09^{* * *}$ & $-2,46 \mathrm{e}-09^{* * *}$ \\
\hline Año $t+3$ & $-0,0186^{* * *}$ & $-0,0040^{* * *}$ & $-6,57 e-09^{* * *}$ & $-7,73 \mathrm{e}-09^{* * *}$ \\
\hline Año $\mathrm{t}+4$ & $-0,0215^{* * *}$ & $-0,0029^{* * *}$ & & \\
\hline LR Chi 2 (7) & $20547,76^{* * *}$ & $46177,28^{* * *}$ & & $132238,10^{* * *}$ \\
\hline LR Chi 2 (6) & & & $132053,42^{* * *}$ & \\
\hline Muestra & 1206 & 1135 & 716 & 716 \\
\hline \multicolumn{5}{|l|}{$* * *$ significativo al $1 \%$} \\
\hline Logit binomial en el panel & \multicolumn{4}{|c|}{ pasa a a la inactividad } \\
\hline Base = sigue como THR & $1998-2002$ & $2002-2006$ & $2007-2010$ & $2007-2010$ \\
\hline Nivel de educación & $-0,0945^{* * *}$ & $-0,1448^{* * *}$ & $-0,0419^{* * *}$ & $-0,0452^{* * *}$ \\
\hline Edad promedio & $0,0298^{* * *}$ & $0,0259^{* * *}$ & $-0,0183^{* * *}$ & $-0,0129^{* * *}$ \\
\hline Jefe de hogar & $-0,4187^{* * *}$ & $-0,6236^{* * *}$ & $-0,4071^{* * *}$ & $-0,4124^{* * *}$ \\
\hline Lengua materna nativa & $\mathrm{n}, \mathrm{d}$ & $\mathrm{n}, \mathrm{d}$ & $\mathrm{n}, \mathrm{d}$ & $0,1493^{* * *}$ \\
\hline Año $t+1$ & $-0,1971^{* * *}$ & $-0,4418^{* * *}$ & $0,1364^{* * *}$ & $0,1227^{* * *}$ \\
\hline Año $t+2$ & $-0,3222^{* * *}$ & $-0,5069^{* * *}$ & $0,1261^{* * *}$ & $0,1166^{* * *}$ \\
\hline Año $t+3$ & $-0,1741^{* * *}$ & $-0,5593^{* * *}$ & $0,0815^{* * *}$ & $0,0599^{* * *}$ \\
\hline Año $t+4$ & $-0,2396^{* * *}$ & $-0,6791^{* * *}$ & & \\
\hline Efectos marginales & $1998-2002$ & $2002-2006$ & $2007-2010$ & $2007-2010$ \\
\hline Nivel de educación & $-0,0231^{* * *}$ & $-0,0359^{* * *}$ & $-0,0086^{* * *}$ & $-0,0113^{* * *}$ \\
\hline Edad promedio & $0,0073^{* * *}$ & $-0,0064^{* * *}$ & $-0,0038^{* * *}$ & $-0,0032^{* * *}$ \\
\hline Jefe de hogar & $-0,1036^{* * *}$ & $-0,1477^{* * *}$ & $-0,0784^{* * *}$ & $-0,0997^{* * *}$ \\
\hline Lengua materna nativa & $\mathrm{n}, \mathrm{d}$ & $\mathrm{n}, \mathrm{d}$ & $\mathrm{n}, \mathrm{d}$ & $0,0369^{* * *}$ \\
\hline Año $t+1$ & $-0,0487^{* * *}$ & $-0,1069^{* * *}$ & $0,0285^{* * *}$ & $0,0305^{* * *}$ \\
\hline Año $t+2$ & $-0,0797^{* * *}$ & $-0,1227^{* * *}$ & $0,0264^{* * *}$ & $0,0289^{* * *}$ \\
\hline Año $t+3$ & $-0,0426^{* * *}$ & $-0,1347^{* * *}$ & $0,0169^{* * *}$ & $0,0149^{* * *}$ \\
\hline Año $t+4$ & $-0,0587^{* * *}$ & $-0,1619^{* * *}$ & & \\
\hline LR Chi 2 (7) & $29828,44^{* * *}$ & $319708,47^{* * *}$ & & $55416,73^{* * *}$ \\
\hline LR Chi 2 (6) & & & $31315,26^{* * *}$ & \\
\hline Muestra & 6547 & 10750 & 6199 & 6199 \\
\hline
\end{tabular}

*** significativo al $1 \%$ 


\section{REFERENCIAS BIBLIOGRÁFICAS}

Blanchard, O., Diamond, P., Hall, R. y J. Yellen (1989). The Beveridge Curve. Brooking Papers on Economic Activity, 1989(1), 1-76. https://doi.org/10.2307/2534495

Bunster, Ximena y Elsa M. Chaney (1985). Sellers and Servants: Working Women in Lima, Peru. Nueva York: Praeger.

Burdett, Kenneth (1978). A Theory of Employee Job Search and Quit Rates. The American Economic Review, 68(1), 212-220.

Deaton, Angus (1997). The Analysis of Household Surveys. A Microeconometric Approach to Development Policy. Washington, D.C.: The World Bank. https://doi.org/10.1596/08018-5254-4

Garavito, Cecilia (2010). Vulnerabilidad en el empleo, género y etnicidad. Economía, XXXIII(65), 89-127. Departamento de Economía de la Pontificia Universidad Católica del Perú.

Garavito, Cecilia (2013). Una mirada al trabajo doméstico remunerado en el siglo XXI, Gestión. Recuperado el 17/11/14 de http://gestion.pe/impresa/trabajo-domestico-remuneradosiglo-xxi-2077485.

Garavito, Cecilia (2015). Evolución del mercado de trabajo doméstico remunerado en el Perú. Documento de Trabajo 407. Departamento de Economía de la Pontificia Universidad Católica del Perú.

Garavito, Cecilia (2016). Trabajadoras del hogar en el Perú y transiciones laborales. Documento de Trabajo 407. Departamento de Economía de la Pontificia Universidad Católica del Perú.

Garavito, Cecilia (2017). «Oferta de trabajo del hogar remunerado en el Perú: 2007-2014». Aceptado para su publicación en un número especial de la Revista Cuadernos de Economía de la Universidad Nacional de Colombia.

Jovanovic, Boyan (1979). Firm-Specific Capital and Turnover. The Journal of Political Economy, 87(6), 1246-1260. https://doi.org/10.1086/260834

Jovanovic, Boyan (1984). Turnover and Unemployment. The Journal of Political Economy, 92(1), 108-122. https://doi.org/10.1086/261210

Kletzer, Lori (1998). Job Displacement. Journal of Economic Perspectives, 12(1), 115-136. https:// doi.org/10.1257/jep.12.1.115

Lockwood, Ben (1991). Information Externalities in the Labour Market and the Duration of Unemployment. The Review of Economic Studies, 58(4), June. https://doi.org/10.2307/ 2297830

Long, J. S. y J. Freese (2014). Regression Models for Categorical Dependent Variables Using Stata. Texas: Stata Press Publication, Statacorp.

Morales, R., J. Rodríguez, M. Higa y R. Montes (2010). Transiciones laborales, reformas estructurales y vulnerabilidad laboral en el Perú (1988-2008). Documento de Trabajo 281. Departamento de Economía de la Pontificia Universidad Católica del Perú.

Mortensen, Dale y Christopher Pissarides (1994). Job Creation and Job Destruction in the Theory of Unemployment. The Review of Economic Studies, 61(3), 397-415. https://doi. org/10.2307/2297896

Mortensen, Dale y Christopher Pissarides (1999). Unemployment Responses to 'Skill-Biased' Technology Shocks: The Role of Labor Market Policy. The Economic Journal, 209(455), 242-265. https://doi.org/10.1111/1468-0297.00431 
Organización Internacional del Trabajo (2013). Trabajo doméstico remunerado en el Perú. Situación y perspectivas en función del Convenio 189 y la Recomendación 201 de la Organización Internacional del Trabajo. Documento de Trabajo. Lima: Organización Internacional del Trabajo. Recuperado el 17/11/14 de http://www.ilo.org/wcmsp5/groups/public/--americas/---ro-lima/documents/publication/wcms_213173.pdf

Rodríguez, José y Gabriel Rodríguez (2012). Movilidad en los mercados laborales del Perú: 2007-2011. En C. Garavito e I. Muñoz (eds.), Empleo y protección social (pp. 239-269). Lima: Fondo Editorial de la Pontificia Universidad Católica del Perú.

Documento recibido el 9 de agosto de 2016 y aprobado el 28 de octubre de 2016 